\title{
High Performance Supercapacitor based on Polypyrrole /Melamine Formaldehyde Resin Derived Carbon Material
}

\author{
Lifang Song ${ }^{1}$, Yongjin Zou ${ }^{2,3,4, *}$, Haitao Zhang ${ }^{2,3,4}$, Cuili Xiang ${ }^{2,3,4}$, Hailiang Chu ${ }^{2,3,4}$, Shujun Qiu $^{2,3,4}$, \\ Erhu Yan ${ }^{2,3,4}$, Fen $\mathrm{Xu}^{2,3,4}$, Lixian Sun ${ }^{2,3,4}$ \\ ${ }^{1}$ School of Materials Science and Engineering, Chang'an University, Xi'an 710061, P.R. China \\ ${ }^{2}$ Guangxi Key Laboratory of Information Materials, Guilin University of Electronic Technology, \\ Guilin 541004, P.R. China \\ ${ }^{3}$ Guangxi Collaborative Innovation Center of Structure and Property for New Energy Materials, Guilin \\ 541004, P.R. China \\ ${ }^{4}$ Guangxi Talent Center of Fundamental and Application for Advanced Functional Materials, Guilin \\ 541004, P.R. China \\ *E-mail: zouy@guet.edu.cn, $\underline{\text { sunlx @ guet.edu.cn }}$
}

doi: $10.20964 / 2017.02 .38$

Received: 7 November 2016 / Accepted: 12 December 2016 / Published: 30 December 2016

In this study, N-doped super-activated carbon material was synthesized using melamine formaldehyde (MF) resin as carbon source and sequent $\mathrm{KOH}$-activation method. Polypyrrole nanofibers (PPy) were further polymerized on the MF resin derived carbon (MFC). The PPy/MFC composite possesses high surface area, which is suitable for high performance supercapacitor electrode materials. Electrochemical measurements indicate the hybrid PPy/MFC electrode yielded the highest specific capacitance of $336.8 \mathrm{~F} \mathrm{~g}^{-1}$ at a current density of $1 \mathrm{~A} \mathrm{~g}^{-1}$ (in $6 \mathrm{M} \mathrm{KOH}$ ). The PPy/MFC electrode also keeps a high capacitance retention ratio of $94.3 \%$ after 3000 cycles. Given the potential massive production and excellent electrochemical performance, The PPy/MFC nanocomposites are promising electrode materials for supercapacitor applications.

Keywords: Supercapacitor; N-doped super-activated carbon; Melamine formaldehyde resin; Polypyrrole, Nanocomposite

\section{$\underline{\text { FULL TEXT }}$}

(C) 2017 The Authors. Published by ESG (www.electrochemsci.org). This article is an open access article distributed under the terms and conditions of the Creative Commons Attribution license (http://creativecommons.org/licenses/by/4.0/). 